\title{
Teddybär-Spital - spielerische Annäherung an eine schwierige Thematik
}

Sarah Schroth

Korrespondenz: Sarah Schroth

Weyermannstrasse 44 CH-3008 Bern
Im Kinderspital Zürich findet am 1. November das erste Treffen des Teddyspitals Zürich statt. Das in Schweden Ende der 1990er Jahre entstandene und seither international erfolgreiche Konzept soll Kinder in spielerischer Atmosphäre an das Thema «Körper, Krankheit und Krankenhaus» heranführen und so die Ängste, die mit dem Arzt- und Spitalbesuch verbunden sind, abbauen. Als kleine Eltern ihrer kranken Stofftiere können die Kinder die Untersuchungen, Behandlungen und Operationen hautnah miterleben und die als Teddyärzte fungierenden Medizinstudenten in ihrer Arbeit unterstützen. Dadurch verlieren die Kinder einen grossen Teil der Skepsis gegenüber der Krankenhausumgebung. Einen Tag mit Spass, Lachen und Freude kann den Kindern und ihren Eltern viele unnötig vergossenen Tränen, Sorgen und Leiden ersparen.

Jeder Arzt und Medizinstudent weiss, welche Herausforderung die Behandlung von Kindern sein kann. Oft reicht schon der Anblick des weissen Kittels, um die Tränen im Schwall über die roten Fieberbäckchen strömen zu lassen. Die Angst der kleinen Patienten ist durchaus nachvollziehbar: Welches kränkelnde Kind verlässt schon gerne sein kuscheliges Bett, um in einem grossen Gebäude von Fremden gestupst und gepikst zu werden. Zusätzlich spürt natürlich auch jedes Kind die Unruhe der besorgten Eltern, für die jeder Spitalbesuch ein besorgniserregendes Ereignis ist. Schon die kleinste Untersuchung kann so für beide Parteien zu einer mühsamen und unangenehmen Prozedur werden. Das Teddybär-Spital schafft hier Abhilfe. Die Kinder werden auf spielerische Weise an verschiedene Untersuchungen und Krankenhausabläufe herangeführt, damit sie die Chance erhalten, das Spital als einen Ort kennenzulernen, wo nicht nur gestochen und in den Bauch gedrückt wird, sondern sie umsorgt werden und ihnen geholfen wird.

\section{Ablauf im Teddybär-Spital}

Behutsam nimmt Anna den Arm ihres Teddys in ihre Kinderhand: «Itz muesch de ganz tapfer si. Es git en chlinä Stich.» Und schwups stupst Teddyarzt Susanne die Spritze gekonnt und zielsicher in den Teddyarm.

Insgesamt dauert der Parcours etwa 1,5 Stunden und beinhaltet sowohl die Besichtigung einer Klinikabteilung, wie auch das Durchlaufen der verschiedenen Stationen des Teddybär-Spitals. Als Vorbereitung werden die Kinder mit Hilfe verschiedene Bücher und

\section{«Hôpital des nounours»}

Le $1^{\text {er }}$ novembre se déroulera pour la première fois à l'hôpital pour enfants de Zurich le projet «Hôpital des nounours». Ce projet, connu et apprécié à l'échelle internationale, a pour objectif de familiariser les petits du jardin d'enfants de façon ludique avec le thème «corps, maladie et hôpital». Les peluches des enfants serviront de patients, qui seront admis par les étudiants en médecine dans «l'hôpital des nounours», pour être examinés et soignés. Le but est de présenter l'hôpital aux enfants comme un univers agréable, où l'on reçoit des soins et de l'aide. Tout comme leurs prédécesseurs à Bâle, Genève ou Lausanne, les étudiants en médecine de Zurich ont investi beaucoup de temps et de travail pour monter ce projet à Zurich. L'idée est de rendre l'hôpital des nounours régulièrement accessible dans l'espace public aux parents et aux enfants intéressés.

Spiele in das Thema eingeführt und können ihrem Teddy ein Symptom, eine Krankheit oder eine Verletzung zuordnen. Der Besuch im Teddyspital beginnt mit der ersten «Anamnese-Station». Dort werden die Teddyeltern zur Krankheit oder Verletzung ihres Stofftieres befragt, bevor der Teddypatient vorsichtig untersucht wird. Danach folgt die «Labor-Station», wo eine Blutabnahme oder Impfung erfolgt. Da dies für die meisten Kinder den schlimmsten Teil des Arztbesuches darstellt, achten die geschulten Studenten hier besonders auf eine sensible und vorsichtige Durchführung. Die Behandlung am dritten Posten besteht - je nach Diagnose - aus einer Röntgen- oder Ultraschalluntersuchung, einem Gips, oder einer Wundversorgung. Zum Abschluss erfolgt der «Apotheken- und Präventionsposten». Hier werden die Kinder $\mathrm{zu}$ verschiedenen Gesundheitsthemen wie z.B. Ernährung, Sonnenschutz oder Mundhygiene aufgeklärt. Abgeschlossen wird die Veranstaltung bei einem gemeinsamen Znüni mit den geheilten und zufriedenen Stofftierpateienten. 


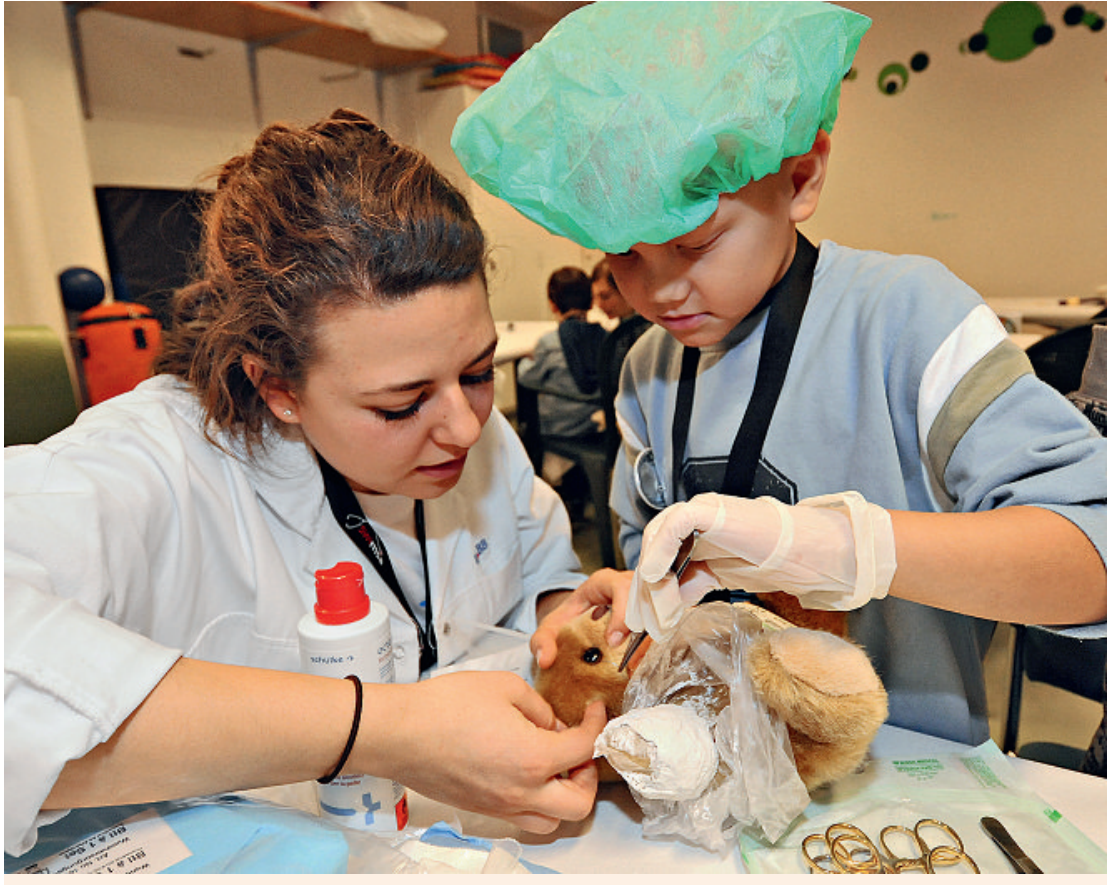

Volle Konzentration: Behandlung eines Patienten im Teddy-Spital.

\section{Teddybär-Spital International}

Seit der Gründung 1999 hat sich das Konzept Teddybär-Spital schweizweit wie auch international durchgesetzt. Organisiert wird das Projekt ehrenamtlich von Medizinstudenten verschiedener Fakultäten, die sehr viel kreative Überlegungen und Zeit in die Projekte investieren. London, Berlin und Schweden sind nur einige Beispiele für die über 30 Länder, die ein zum Teil seit Jahren bestehendes «Teddy Bear Hospital» in regelmässigen Abständen anbieten. Dieses Projekt hat seinen Ursprung in der IFMSA (International Federation of Medical Students' Association). Motivierte Medizinstudenten brachten dieses Projekt anschliessend in die swimsa (Swiss Medical Student Association). Seither unterstützt die swimsa, u.a. durch Verteilung von Flyern und öffentliche Stände, die Teddybärspitäler in der ganzen Schweiz.

Der Erfolg der Einführung des Teddybär-Spitals in Fribourg und Basel sprach sich schnell herum. Unter anderem aufgrund mehrerer Anfragen von Zürcher Kindergärten wurde das Projekt nun auch nach Zürich geholt. In Zusammenarbeit mit dem Kinderspital Zürich und lokalen Kindergärten hat sich eine Gruppe von engagierten Medizinstudenten der Universität Zürich zusammengeschlossen, um am 1. November das Projekt zu lancieren. An diesem Tag werden vier Kindergartenklassen das gesamte Programm des Teddybär-Spitals erleben dürfen. Schirmherr und aktiver Mitorganisator des Projektes ist Dr. Sepp Holtz, Facharzt für Kinder- $u$. Jugendmedizin FMH, der auch für einen Teil der «Ausbildung» der freiwilligen Teddydoktoren sorgt.

\section{Zukunftsprojekte und Ziele}

Nach dem sehr positiven Feedback der Basler und Lausanner Kindergärten findet das Projekt mittlerweile durch die Unterstützung verschiedener Sponsoren (u.a. VSAO, Mundipharma, Spirig, Mepha, SGAM, fymed u.a.) mehrmals pro Jahr statt. Ziel ist es, auch in Zürich regelmässige Teddybär-Spitalbesuche mit den dortigen Kindergärten organisieren zu können. Läuft das Projekt in Zusammenarbeit mit den Kindergärten erfolgreich an, könnte es im Rahmen einer öffentlichen Veranstaltung auch allen interessierten Eltern und Kindern zugänglich gemacht werden.

Zusätzlich ist in Zürich eine Erweiterung auf Grundschulebene geplant. Das Projekt mit dem Namen «Flying Dr. Ted» soll für Kinder ab 9 Jahren in verschiedenen Grundschulen angeboten werden. Dabei gewähren Medizinstudenten den Kindern einen Einblick in die Prinzipien des Arztberufes und der Krankheitslehre. Welche häufigen Krankheiten gibt es? Wie werden diese behandelt, und wie kann man sich davor schützen? Ähnlich wie im TeddybärSpital denken die Kinder sich verschiedene Krankheiten für ihre Stofftiere oder Puppen aus, die in der Gruppe miteinander besprochen werden. Hier haben die Kinder auch die Chance, in die Rolle des Arztes zu schlüpfen und verschiedene Instrumente, wie Stethoskop und Reflexhammer kennenzulernen.

Das Projekt Flying Dr. Ted hat neben der Annäherung der Kinder an das Spital den Anspruch, das Gesundheitsbewusstsein der Kinder $\mathrm{zu}$ fördern. Mit Spielen, Theater und interaktiven Präsentationen bringen die Studenten den Kindern die Grundlagen gesunder Ernährung und körperlicher Aktivität bei. In Zusammenarbeit mit Studenten der Zahnmedizin ist auch geplant, den Kindern die Anatomie und Prinzipien der Zahnhygiene nahezubringen.

\section{Mithilfe und Sponsoring}

Dank des Engagements der motivierten Studenten konnte das Projekt in vielen ehrenamtlichen Arbeitsstunden zur Vorbereitung und Planung ins Leben gerufen werden. Dadurch beschränken sich die Kosten auf Materialien, Logistik und Raummiete. Das Sponsoring deckt einen Grossteil der bisherigen Kosten. Damit das Projekt auch langfristig erhalten und ausgebaut werden kann, hoffen die Organisatoren auf weitere Mithilfe und Unterstützung. Kinder sind die Zukunft! Wem die Prävention von Krankheiten und Unfällen ebenso ein Anliegen ist wie eine für Kinder gleichermassen angenehme und effiziente medizinische Behandlung, bekommt in diesem Projekt die Chance, einen sinnvollen Beitrag zu leisten. 